\title{
Erratum to: Burkholderia pseudomallei induces IL-23 production in primary human monocytes
}

\author{
Panthong Kulsantiwong ${ }^{1}$ Matsayapan Pudla ${ }^{2} \cdot$ Jitrada Boondit $^{1}$. \\ Chanthiwa Wikraiphat $^{3}$ - Susanna J. Dunachie ${ }^{3}$ - Narisara Chantratita ${ }^{3,4}$. \\ Pongsak Utaisincharoen ${ }^{1}$
}

Published online: 5 April 2016

(C) Springer-Verlag Berlin Heidelberg 2016

\section{Erratum to: Med Microbiol Immunol DOI 10.1007/s00430-015-0440-z}

The original version of this article unfortunately contained a mistake. The labelling of Fig. 1 was incorrect. The correct symbols are black triangle for wild type (1026b) and black square for LPS mutant (SRM117) as shown in Fig. 1 below.

However, the discussion in the original version of this article is not affected.
The online version of the original article can be found under doi:10.1007/s00430-015-0440-z

Pongsak Utaisincharoen

pongsak.uta@mahidol.ac.th

1 Department of Microbiology, Faculty of Science, Mahidol University, Bangkok, Thailand

2 Department of Oral Microbiology, Faculty of Dentistry, Mahidol University, Bangkok, Thailand

3 Mahidol-Oxford Tropical Medicine Research Unit, Faculty of Tropical Medicine, Mahidol University, Bangkok, Thailand

4 Department of Microbiology and Immunology, Faculty of Tropical Medicine, Mahidol University, Bangkok, Thailand

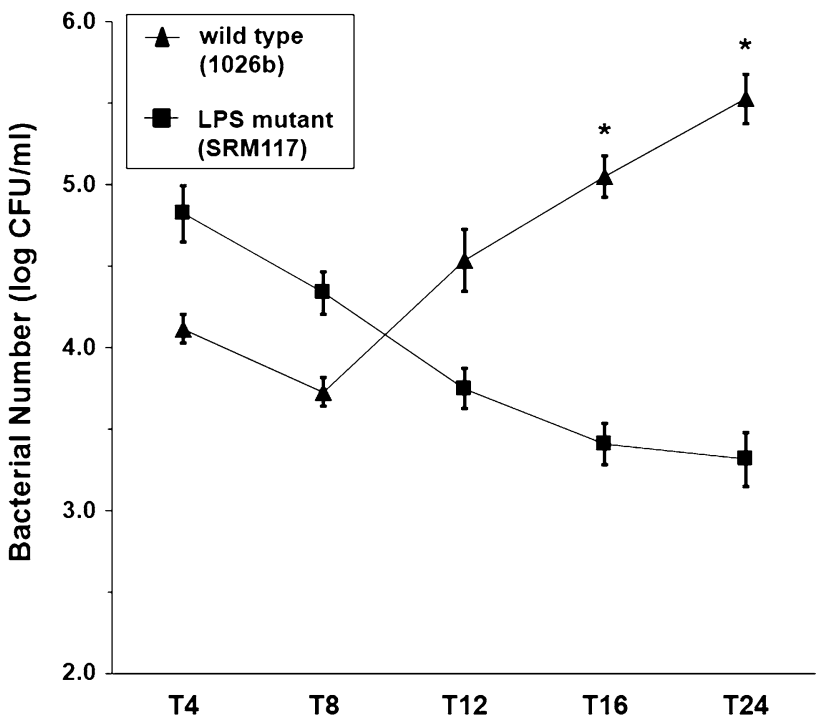

Fig. 1 Survival kinetics of wild-type B. pseudomallei (1026b) and B. pseudomallei LPS mutant (SRM117) inside primary human monocytes at different time points. Primary human monocytes were infected with each bacteria strain at MOI of 1:1 by antibiotic protection assay. Primary human monocytes viability was determined using $0.04 \%$ trypan blue staining to ensure that around $80 \%$ of host cells remain alive at the final time point. The results represent mean $\mathrm{CFU} \pm \mathrm{SE}$ of seven independent experiments. Data from seven independent experiments were analyzed by Student's $t$ test $\left({ }^{*} p<0.05\right)$ 